\title{
Response prediction to oxaliplatin plus 5-fluorouracil chemotherapy in patients with colorectal cancer using a four-protein immunohistochemical model
}

\author{
JUNJIE GU ${ }^{1}$, ZHE LI $^{1}$, JIANFENG ZHOU ${ }^{2}$, ZHAO SUN $^{2}$ and CHUNMEI BAI ${ }^{2}$ \\ ${ }^{1}$ Department of Medical Oncology, Peking Union Medical College Hospital, \\ Chinese Academy of Medical Sciences and Peking Union Medical College; \\ ${ }^{2}$ Department of Medical Oncology, Peking Union Medical College Hospital, \\ Chinese Academy of Medical Sciences, Dongcheng, Beijing 100730, P.R. China
}

Received August 9, 2018; Accepted April 29, 2019

DOI: $10.3892 / \mathrm{ol} .2019 .10474$

\begin{abstract}
The response of cancer patients to oxaliplatin combined with 5-fluorouracil (5-FU) is difficult to predict. It has been reported that carcinoma-associated fibroblasts (CAFs) could induce AKT and ERK phosphorylation, and upregulate survivin expression in colorectal cancer (CRC) cells, which could lead to oxaliplatin plus 5-FU resistance. A total of 71 patients with advanced CRC (aCRC) treated with oxaliplatin plus 5-FU were included in the present study. These patients comprised 46 chemotherapy responders and 25 non-responders. The expression levels of $\alpha$-smooth muscle actin ( $\alpha$-SMA), phosphorylated (p)-AKT, p-ERK and survivin were determined by immunohistochemical evaluation of paraffin-embedded samples from patients. A predictive model was established using a Probabilistic Neural Network model. The high expression of $\alpha$-SMA, p-AKT and survivin in patients with aCRC were associated with oxaliplatin plus 5-FU resistance $(\mathrm{P}<0.001, \mathrm{P}=0.023$ and $\mathrm{P}=0.001$, respectively). Furthermore, patients with stage IV CRC exhibiting high expression levels of $\alpha$-SMA and survivin experienced a reduced progression-free survival time compared with patients with low expressions of $\alpha$-SMA and survivin (5.5 vs. 15.0 months; 5.5 vs. 15.0 months; $\mathrm{P}=0.005$ and $\mathrm{P}=0.001$, respectively). Stage IV CRC and high survivin expression predicted a reduced overall survival time compared with that for patients with stage IV CRC and low survivin expression
\end{abstract}

Correspondence to: Professor Zhao Sun or Professor Chunmei Bai, Department of Medical Oncology, Peking Union Medical College Hospital, Chinese Academy of Medical Sciences, 1 Shuaifuyuan, Dongcheng, Beijing 100730, P.R. China

E-mail: jessiesz@126.com

E-mail: baichunmei1964@163.com

Key words: predictive model, oxaliplatin plus 5-fluorouracil, response, advanced colorectal cancer, intrinsic resistance
(50.0 vs. 15.0 months; $\mathrm{P}<0.001$ ). Patients with $\alpha$-SMA, p-AKT, p-ERK and survivin overexpression were more likely to present with intrinsic resistance to the oxaliplatin plus 5-FU regimen (the accuracies of modeling, validation and prediction were $83.7,92.9$ and $85.7 \%$, respectively). In conclusion, the multifactorial predictive biomarker model of $\alpha$-SMA, p-AKT, p-ERK and survivin expression for patients with aCRC to predict intrinsic resistance to oxaliplatin plus 5-FU regimens is of great efficiency and accuracy. Patients with high expression of this predictive model may be intrinsically resistant to the oxaliplatin and 5-FU regimen.

\section{Introduction}

Colorectal cancer (CRC) is the third most commonly diagnosed cancer and the leading cause of cancer-associated mortality worldwide (1). Traditional chemotherapy remains one of the standard treatments of CRC. In particular, oxaliplatin plus 5-fluorouracil (5-FU)-based regimens, also known as FOLFOX, XELOX and SOX, are the first-line chemotherapy regimens for patients with advanced CRC (aCRC); however, the objective response rate of this regimen is estimated to be $\sim 50 \%$ (2). In addition, numerous patients with CRC cannot benefit from chemotherapy due to intrinsic or acquired chemotherapy resistance (3). It is therefore difficult for oncologists to select the most suitable regimens for patients with CRC. Determining effective and distinct biomarkers to characterize patients who are intrinsically resistant to oxaliplatin plus 5-FU regimens would have a significant influence in clinical practice.

Several tumor molecular markers that can predict the efficacy of 5-FU or oxaliplatin have been reported, including DNA damage repair system genes, auch as X-ray repair cross complementing 1 (4), ERCC excision repair 1, endonuclease non-catalytic subunit (5) and ERCC excision repair 5 endonuclease (6), certain key enzymes in the chemotherapy-associated signaling pathway, such as thymidylate synthase (7), and other molecules, such as NKx2-3 and transforming growth factor- $\beta 1$-induced transcript 1 (8), homeobox B8 and kallikrein related-peptidase 11 (9). However, the predictive value 
of a single molecular marker is limited due to the participation of multiple genes and proteins, rather than a single gene or protein, in the process of drug resistance. It is therefore crucial to detect multiple genes and proteins, and establish a predictive model that could determine the efficacy and accuracy of the oxaliplatin plus 5-FU regimen in patients with aCRC rather than using the predictive value of one molecular marker only (10).

Previous studies reported that carcinoma-associated fibroblasts (CAFs) are a pivotal part of the tumor microenvironment and serve a crucial role in tumor drug resistance. Gonçalves-Ribeiro et al (11) reported that CAFs have a protective effect on CRC cells and could be associated with chemotherapy resistance in patients with CRC. Furthermore, CAFs can induce the translocation of AKT, survivin and ERK to the nucleus of CRC cells, induce AKT and ERK phosphorylation, and upregulate survivin expression. These phenomena ensure correct DNA repair and accurate cell entrance and exit from mitosis in the presence of chemotherapy. Oxaliplatin plus 5-FU regimen resistance is subsequently induced via the activation of the PI3K/AKT, MAPK and Janus kinase/signal transducer and activator of transcription (JAK-STAT) signaling pathways. In addition, it has been reported that the MAPK (12-14) and PI3K/AKT/mTOR (15-18) pathways serve key roles in drug resistance, notably in CRC, and that PI3K/AKT signaling pathway inhibition can reduce resistance to chemotherapeutic drugs. It was also reported that survivin overexpression, which may be a downstream effect of the MAPK or PI3K-AKT-mTOR signaling pathway (19), is associated with drug resistance in CRC. As all these findings focus on cellular and molecular approaches, it is essential to verify whether they exist in patients with CRC. The present study therefore investigated the association between $\alpha$-SMA, p-AKT, p-ERK and survivin expression and oxaliplatin plus 5 -FU chemotherapy efficacy in patients with aCRC. Since chemotherapy resistance is a multifactorial process, the present study aimed to establish a predictive model that could help oncologists to screen patients with intrinsic resistance to chemotherapeutic drugs.

\section{Materials and methods}

Patients and tissue samples. A total of 71 patients diagnosed with aCRC at the Peking Union Medical College Hospital (Beijing, China) between June 2013 and February 2018 were enrolled in the present study. Tissue samples were obtained from patients following radical CRC resection or biopsy during colonoscopy. All patients provided written informed consent. The inclusion criteria were as follows: i) All patients were histologically diagnosed with aCRC and cancer stage was evaluated according to the American Joint Committee on Cancer, 7th edition (20); ii) all patients received oxaliplatin plus 5-FU regimens in accordance with the National Comprehensive Cancer Network guideline (21), including mFOLFOX6, which consisted of $85 \mathrm{mg} / \mathrm{m}^{2}$ oxaliplatin on day $1,400 \mathrm{mg} / \mathrm{m}^{2}$ leucovorin on day $1,400 \mathrm{mg} / \mathrm{m}^{2}$ intravenous (IV) bolus 5 -FU on day 1 , and then $1,200 \mathrm{mg} / \mathrm{m}^{2} /$ day for $48 \mathrm{~h}$ by continuous IV infusion, repeating every 2 weeks and evaluated every 4 cycles using the Response Evaluation Criteria in Solid Tumors (RECIST 1.1) (22), or XELOX, which consists of $130 \mathrm{mg} / \mathrm{m}^{2} \mathrm{IV}$ oxaliplatin on day 1 and $1,000 \mathrm{mg} / \mathrm{m}^{2}$ oral capecitabine twice daily for 14 days, repeating every 3 weeks and evaluated every 2-3 cycles using RECIST; iii) non-responders to chemotherapy (NRCs) included patients with stage IV CRC who received first-line chemotherapy and were identified as having progressive disease when evaluated the first time (after 4 cycles of mFOLFOX6 or 2-3 cycles of XELOX), and patients with stage III CRC who received adjuvant chemotherapy following surgery and who relapsed within 6 months. Chemotherapy responders (CRs) referred to patients with stage IV CRC who received first-line chemotherapy and who were evaluated with partial regression or complete regression at most. The chemotherapy responses were evaluated independently in all patients by two oncologists using RECIST. If their conclusions were different, a third oncologist independently evaluated the case, and the three oncologists consulted and obtained the final result together. Patients with CRC who were identified as stable after chemotherapy or as progressive disease following the first evaluation were excluded from the present study.

Immunohistochemistry (IHC) staining and scoring. IHC staining was performed on $4-\mu \mathrm{m}$ thick sections of paraffin-embedded tissue samples to detect the protein expression levels of $\alpha$-SMA, p-AKT, p-ERK and survivin. Briefly, sections were deparaffinized with xylene, rehydrated in decreasing gradient of alcohol and immersed in distilled water for $5 \mathrm{~min}$. Sections were then incubated with EDTA antigen retrieval buffer ( $\mathrm{pH} \mathrm{8.0)}$ in a microwave for $15 \mathrm{~min}$. Sections were treated with $3 \%$ hydrogen peroxide for $25 \mathrm{~min}$ at room temperature in the dark to block endogenous peroxidase activity, washed with PBS three times for 5 min and blocked with $3 \%$ bovine serum albumin at room temperature for $30 \mathrm{~min}$. Sections were incubated with the following primary antibodies at $4^{\circ} \mathrm{C}$ overnight: Mouse anti-human $\alpha$-SMA (cat. no. GB13044, 1:1,000; Servicebio), mouse anti-human p-ERK (cat. no. GB13004; 1:1,000; Servicebio), rabbit anti-human p-AKT (cat. no. GB13012-3; 1:100; Servicebio) and rabbit anti-human survivin (cat. no. 10508-1-AP; 1:100; ProteinTech Group, Inc.). Sections were then incubated with goat anti-mouse secondary antibody (cat. no. GB23301; 1:200; Servicebio; Startech) or goat anti-rabbit secondary antibody (cat. no. GB13044; 1:1,000; Servicebio; Startech) at room temperature for $50 \mathrm{~min}$. Sections were developed using 3,3'-diaminobenzidine and counterstained with hematoxylin at room temperature for $5 \mathrm{~min}$. Images were captured under light microscopy.

All slides were independently evaluated by two pathologists who had no knowledge of the clinical information. If their conclusions were different, a third pathologist independently evaluated the case, and the three pathologists discussed and obtained the final result together. Positive $\alpha$-SMA expression was localized in the cytoplasm of CAFs; positive survivin expression was localized in the cytoplasm of CRC cells; positive p-AKT and p-ERK expression was localized in the nucleus of CRC cells due to AKT and ERK nuclear translocation. The staining intensity of $\alpha$-SMA expression was visually scored and classified as follows: 0 , no staining; 1 , light brown staining; and 2, brown staining. The proportion of cells positively stained for $\alpha$-SMA was graded as follows: 
0 , no positively stained CAFs/total CAFs in high-power fields (HPFs); 1, positively stained CAFs/total CAFs in HPFs $<10 \%$; 2 , positively stained CAFs/total CAFs in HPFs between 10 and $50 \%$; and 3 , positively stained CAFs/total CAFs in HPFs $>50 \%$. The staining intensity of p-AKT, p-ERK and survivin was visually scored and classified as follows: 0 , no staining; 1 , light brown staining; and 2, brown staining. The proportion of cells positively stained for $\mathrm{p}-\mathrm{AKT}$, $\mathrm{p}-\mathrm{ERK}$ or survivin was graded as follows: 0 , no positively stained CRC cells/total CRC cells in HPFs; 1, positively stained CRC cells/total CRC cells in HPFs $<10 \% ; 2$, positively stained CRC cells/total CRC cells in HPFs between 10 and 50\%; 3, positively stained CRC cells/total CRC cells in HPFs $>50 \%$. The staining index (SI) of $\alpha$-SMA, p-AKT, p-ERK and survivin was calculated as follows: $\mathrm{SI}=$ staining intensity $\mathrm{x}$ proportion of positive cells. The results were scored from 0 to 6 . Low expression was defined as an SI of 0 to 2 and high expression was defined as an SI of 3 to 6 (23).

Follow-up of patient survival. Follow-ups were designed to assess the progression-free survival (PFS), disease-free survival (DFS) and overall survival (OS) of the patients. The latest follow-up of the patients was performed on February 28, 2019.

\section{Data analysis, modeling and validation}

Analysis of data characteristics. Expression levels of $\alpha$-SMA, p-AKT, survivin and p-ERK were used as features for this model and scored as 1 or 2 (low and high expression, respectively). First, the accuracy of these four features as predictive biomarkers for chemotherapy efficiency in patients with aCRC who were identified as NRCs or CRs was analyzed. Subsequently, the two features of highest accuracy were selected, integrated and their accuracy was analyzed. The selection was then increased to three and four features in order to analyze the predictive accuracy.

Probabilistic Neural Networks (PNN) model. An artificial neural network is a type of artificial intelligence; it has been recently widely used in numerous fields, including clinical diagnosis, screening and prognosis prediction (24). As PNNs use the advantages of a radial basis network and classical probability density estimation theory, they offer particularly significant advantages in pattern classification. The PNN classification was therefore used in the present study as the model for classification problem.

The MATLAB Neural Network tool cabinet provides the function 'newpnn' to create probabilistic neural networks. Assuming that the input vector is $\mathrm{P}$ and the target is $\mathrm{T}$, a two-layer neural network named 'net' can be created by using net = newpnn (P, T, SPREAD), where $\mathrm{P}$ is a matrix of $R$ feature quantities of the input vector of the $Q$ group, $R$ rows and $\mathrm{Q}$ columns, $\mathrm{T}$ is a target matrix of $\mathrm{S}$ rows and $\mathrm{Q}$ columns composed of $\mathrm{Q}$ group classification vectors, $\mathrm{S}$ is a classification number, and $\mathrm{S}$ is 2 if it is a class 2 problem. If the sample of the Jth column belongs to the class i, the element of the Jth column of the ith row of the matrix $\mathrm{T}$ is 1 , and the remaining columns of the row are $\sim 0$. SPREAD is the spreading coefficient of the Gaussian radial basis function. The default value is 0.1 . If the SPREAD value is $\sim 0$, the created probabilistic neural network can be used as the nearest neighbor classifier.
The MATLAB tool cabinet provides the function $\mathrm{y}=\mathrm{sim}$ (net, q) by using the probabilistic neural network model created by newpnn, which predicts the target value $y$ of the input vector q using the sim function. This function can therefore be used to verify and check the network model.

The MATLAB tool cabinet also provides the function plotconfusion $(\mathrm{y}, \mathrm{T})$, where $\mathrm{y}$ is the target output value predicted by the network model and $\mathrm{T}$ is the known target value for comparison. In case the target value has only two categories and the function calculates the predictive values as 0 and 1, this function will calculate the number of correct samples and false samples, the accuracy and the error rate.

By using the three functions aforementioned provided by the MATLAB tool cabinet, the classification problem of predicting the target value of 1 (NRCs) or 0 (CRs) based on the four eigenvalues of the provided cases can be accomplished.

The data from the 71 patients were first divided into two parts as follows: 43 patients were used for modeling and the remaining 28 were used for prediction. The data from the 43 patients were divided into two parts by $1,3,5 \ldots, 2,4,6 \ldots$, each of which is 22 lines and 21 lines, and the 22 lines were then further interlaced to obtain two lines of 11, 11 of which were combined with 21 rows into a 32-line dataset $74.4 \%$ of the total samples) for modeling, and the remaining 11 patients ( $25.6 \%$ of the total samples) were used for model validation.

Statistical analysis. The clinical and follow-up data were analyzed using SPSS 24.0 (IBM Corp.). The $\chi^{2}$ test and Fisher's exact test were used to assess the association between patient clinical characteristics and the expression of $\alpha$-SMA, p-AKT, p-ERK and survivin. $\mathrm{P}<0.05$ was considered to indicate a statistically significant difference.

\section{Results}

Patient general characteristics. A total of 71 patients with pathologically confirmed aCRC who received oxaliplatin plus 5-FU chemotherapy were enrolled in the present study. Amongst them, 26 patients were defined as NRCs and 45 patients were defined as CRs (Table I). There were 38 men and 33 women, and the median age of these patients was 62 years old. There was no significant difference between pathology type or primary tumor location and the chemotherapy response $(\mathrm{P}=0.144$ and $\mathrm{P}=0.853$, respectively). A flowchart describing the study design is presented in Fig. 1.

Association between $\alpha-S M A, p-A K T, p-E R K$ and survivin expression and the chemotherapy response. The expression of $\alpha$-SMA, p-AKT, p-ERK and survivin was analyzed by IHC in paraffin-embedded specimens from the patients (Fig. 2). The results detailed in Table I demonstrated that the high expression levels of $\alpha$-SMA, survivin and p-AKT in patients with $\mathrm{CRC}$ were associated with oxaliplatin plus 5-FU resistance (NCRs; $\mathrm{P}<0.001, \mathrm{P}<0.001$ and $\mathrm{P}=0.023$, respectively). There was no significant difference between $\mathrm{p}$-ERK expression and chemotherapy response $(\mathrm{P}=0.227)$.

Association between $\alpha$-SMA, p-AKT, p-ERK and survivin expression and patient PFS, DFS and OS. Among the 71 patients, there were 61 patients (NRCs:CR ratio, 16:45) with stage IV 
Table I. Clinical and pathological characteristics of the 71 patients with colorectal cancer who received oxaliplatin plus 5 -fluorouracil chemotherapy and were classified as responders $(n=46)$ and non-responders $(n=25)$.

\begin{tabular}{|c|c|c|c|c|}
\hline Patient characteristics & $\begin{array}{c}\text { Number of } \\
\text { patients }\end{array}$ & $\begin{array}{l}\text { Chemotherapy } \\
\text { responders }\end{array}$ & $\begin{array}{l}\text { Non-responders to } \\
\text { chemotherapy }\end{array}$ & P-value \\
\hline \multicolumn{5}{|l|}{ Sex, n (\%) } \\
\hline Male & $38(53.5)$ & $23(50.0)$ & $15(60.0)$ & \multirow[t]{2}{*}{0.701} \\
\hline Female & $33(46.5)$ & $23(50.0)$ & $10(40.0)$ & \\
\hline Mean age, years & 62 & 62 & 62.5 & 0.163 \\
\hline \multicolumn{5}{|l|}{ Pathological type, n (\%) } \\
\hline Highly differentiated & 4 (5.6) & 1 (2.2) & $3(12.0)$ & \multirow[t]{4}{*}{0.144} \\
\hline Middle differentiated & $52(73.2)$ & $34(73.9)$ & $18(72.0)$ & \\
\hline Poorly differentiated & $10(14.1)$ & $7(15.2)$ & $3(12.0)$ & \\
\hline Undifferentiated & $5(7.0)$ & $4(8.7)$ & $1(4.0)$ & \\
\hline \multicolumn{5}{|l|}{ Primary location, n (\%) } \\
\hline Left colonic carcinoma & $51(71.8)$ & $33(71.7)$ & $18(72.0)$ & \multirow[t]{3}{*}{0.853} \\
\hline Right colonic carcinoma & $17(23.9)$ & $11(23.9)$ & $6(24.0)$ & \\
\hline Left and right colonic carcinoma & $3(4.2)$ & $2(4.3)$ & $1(4.0)$ & \\
\hline \multicolumn{5}{|l|}{$\alpha$-SMA expression, $\mathrm{n}(\%)$} \\
\hline High & $36(50.7)$ & $15(32.6)$ & $21(84.0)$ & \multirow[t]{2}{*}{$<0.001$} \\
\hline Low & $35(49.3)$ & $31(67.4)$ & $4(16.0)$ & \\
\hline \multicolumn{5}{|l|}{ p-AKT expression, $\mathrm{n}(\%)$} \\
\hline High & $22(31.0)$ & $10(21.7)$ & $12(48.0)$ & \multirow[t]{2}{*}{0.023} \\
\hline Low & $49(69.0)$ & $36(78.3)$ & $13(52.0)$ & \\
\hline \multicolumn{5}{|l|}{ Survivin expression, n (\%) } \\
\hline High & $36(50.7)$ & $16(34.8)$ & $20(80.0)$ & \multirow[t]{2}{*}{$<0.001$} \\
\hline Low & $35(49.3)$ & $30(65.2)$ & $5(20.0)$ & \\
\hline \multicolumn{5}{|l|}{ p-ERK expression, $\mathrm{n}(\%)$} \\
\hline High & $23(32.4)$ & $13(28.3)$ & $10(40.0)$ & \multirow[t]{2}{*}{0.227} \\
\hline Low & $48(67.6)$ & $33(71.7)$ & $15(60.0)$ & \\
\hline
\end{tabular}

$\alpha$-SMA, $\alpha$-smooth muscle actin; p-, phosphorylated.

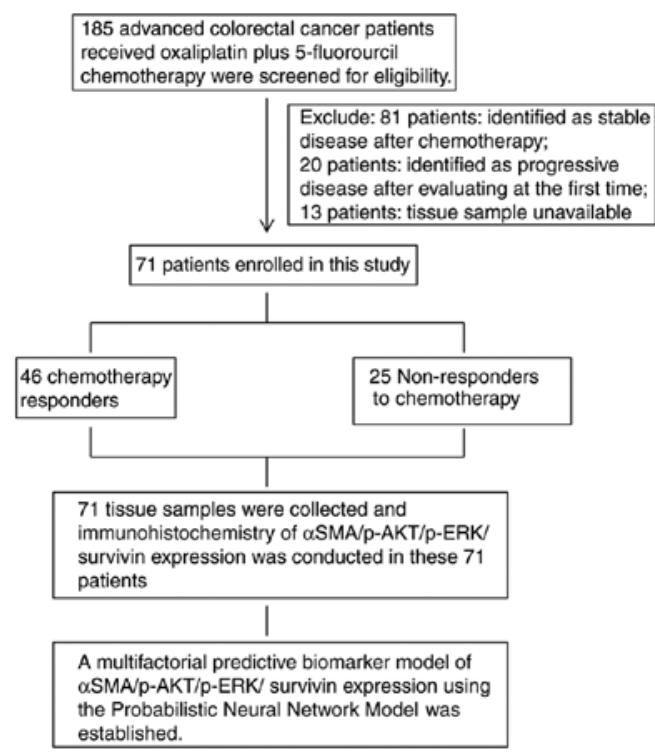

Figure 1. Flowchart describing the study design. Of the 185 patients screened for eligibility, 71 patients were enrolled in the present study, including 46 chemotherapy responders and 25 non-responders to chemotherapy. $\alpha$-SMA, $\alpha$-smooth muscle actin; p-, phosphorylated.
CRC receiving oxaliplatin and 5-FU as first-line chemotherapy, and 10 patients (NRC:CR ratio, 9:1) with stage III with CRC receiving oxaliplatin and 5-FU as adjuvant chemotherapy. Since stage IV patients with CRC and stage III patients with CRC had different prognoses, the association between PFS, DFS and OS and $\alpha$-SMA, p-AKT, p-ERK and survivin expression for these patients were separately analyzed using Kaplan-Meier curves.

The results presented in Figs. 3 and 4 demonstrated that in patients with stage IV CRC, the high expression of $\alpha$-SMA was associated with a significantly reduced PFS time (5.5 vs. 15.0 months; $\mathrm{P}=0.005$; Fig. $3 \mathrm{~A}$ ), and although a shorter $\mathrm{OS}$ time was observed ( 30.7 vs. 35.0 months; $\mathrm{P}=0.218$; Fig. $4 \mathrm{~A}$ ), this difference was not statistically significant. Furthermore, high survivin expression was associated with a significantly reduced PFS time (5.5 vs. 15.0 months; $\mathrm{P}=0.001$; Fig. $3 \mathrm{C}$ ) and a significantly reduced $\mathrm{OS}$ time (15.0 vs. 50.0 months; $\mathrm{P}<0.001$; Fig. $4 \mathrm{C}$ ). There was no significant association between $\mathrm{p}$-AKT and p-ERK expression and PFS (6.0 vs. 11.0 months and 6.0 vs. 11.0 months; $\mathrm{P}=0.516$ and $\mathrm{P}=0.466$, respectively; Fig. $3 \mathrm{~B}$ and $\mathrm{D})$ or $\mathrm{OS}$ (26.7 vs. 35.0 months and 32.7 vs. 35.0 months; $P=0.429$ and $\mathrm{P}=0.446$, respectively; Fig. 4B and D) times. 


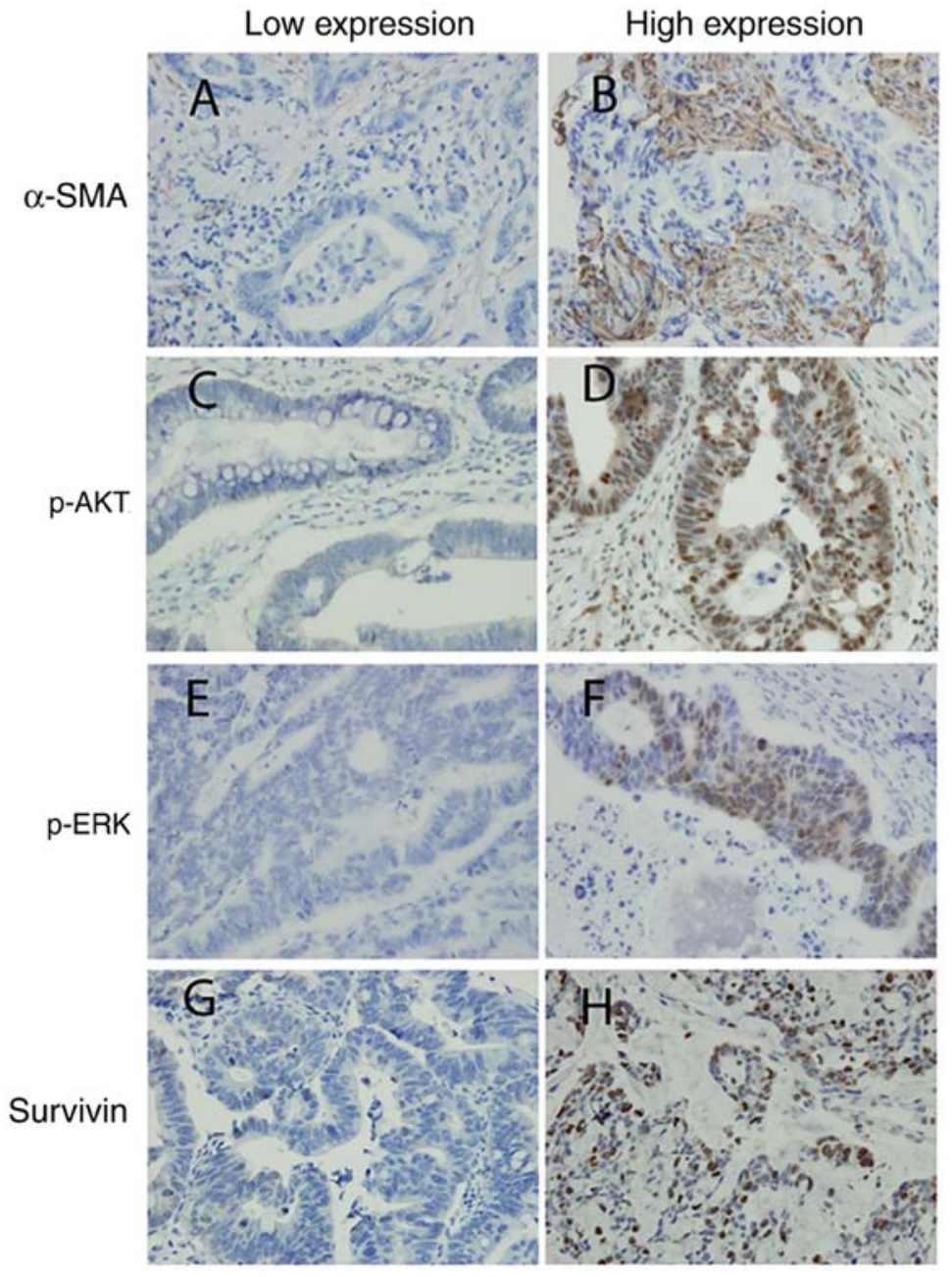

Figure 2. Immunohistochemistry staining for $\alpha$-SMA, p-AKT, p-ERK and survivin. (A) Low $\alpha$-SMA expression and an SI value of 1 (1x1). (B) High $\alpha$-SMA expression and an SI of is 6 (2x3). (C) Low p-AKT expression and an SI of 0 (0x0). (D) High p-AKT expression and an SI of 6 (2x3). (E) Low p-ERK expression and an SI of 0 (0x0). (F) High p-ERK expression and an SI of 6 (2x3). (G) Low Survivin expression and an SI of 0 (0x0). (H) High Survivin expression and an SI of 6 (2x3). The numbers into brackets represent the staining intensity $x$ the proportion of positive cells; low expression was defined as an SI of 0 to 2 and high expression was defined as an SI of 3 to 6. Magnification, x400. $\alpha$-SMA, $\alpha$-smooth muscle actin; p-, phosphorylated; SI, staining index.

The results presented in Fig. 5 demonstrated that in patients with stage III CRC, there was no significant association between $\alpha$-SMA, p-AKT, survivin and p-ERK expression and PFS time (6.2 vs. 6.6 months, 5.0 vs. 6.6 months, 6.6 vs. 3.0 months and 5.0 vs. 6.6 months; $\mathrm{P}=0.949, \mathrm{P}=0.158, \mathrm{P}=0.427$ and $\mathrm{P}=0.280$, respectively; Fig. 5A-D). The results from Fig. 6 also revealed that in patients with stage III CRC, there was no significant association between p-AKT, survivin and p-ERK expression and OS time (15.0 vs. 31.0 months, 24.0 vs. 38.0 months and 12.0 vs. 31.0 months; $\mathrm{P}=0.207, \mathrm{P}=0.264$ and $\mathrm{P}=0.353$, respectively; Fig. 6A-C). In patients with stage III CRC, only 1 patient had high $\alpha$-SMA expression (Fig. 6D) and since this patient was still alive during the study, the association between $\alpha-S M A$ expression and median OS could therefore not be determined.

Predictive model of $\alpha-S M A, p-A K T, p-E R K$ and survivin expression for oxaliplatin plus 5-FU chemotherapy response in patients with $a C R C$. The error rates of $\alpha$-SMA, p-AKT, survivin and p-ERK expressions were first analyzed as predictive biomarkers for chemotherapy response in the 43 patients involved in this model. The error rates of individual feature were 28, 35 and $30 \%$ for $\alpha$-SMA, p-AKT and survivin expression, whereas p-ERK expression did not have discriminating ability (Table II) and was not satisfactory for predicting chemotherapy response. Two features with the lowest error rate, $\alpha$-SMA and survivin expression, were selected from the four features and were used to detect the predictive accuracy of the integrated model. The error rate of the combined model was $26 \%$ (Table III). When p-AKT expression was added to this model, the error rate decreased to $21 \%$ (Table IV). When p-ERK expression was added to this model, the error rate decreased to $16 \%$ and the accuracy was $>80 \%$ (Table V).

Based on these results, the PNN module of the MATLAB tool cabinet was used to establish the model regarding the classification problems. The data from 32 patients were used for modeling, the data from 11 patients were used for validation and the data from 28 patients were used for prediction. The results of modeling, validation and prediction are presented in Table VI. The results demonstrated that the modeling accuracy involving a single feature was $\sim 70 \%$. When the features number increased to 4 , the modeling accuracy was $83.7 \%$, the validation accuracy was $92.9 \%$ and the prediction accuracy was $85.7 \%$. 

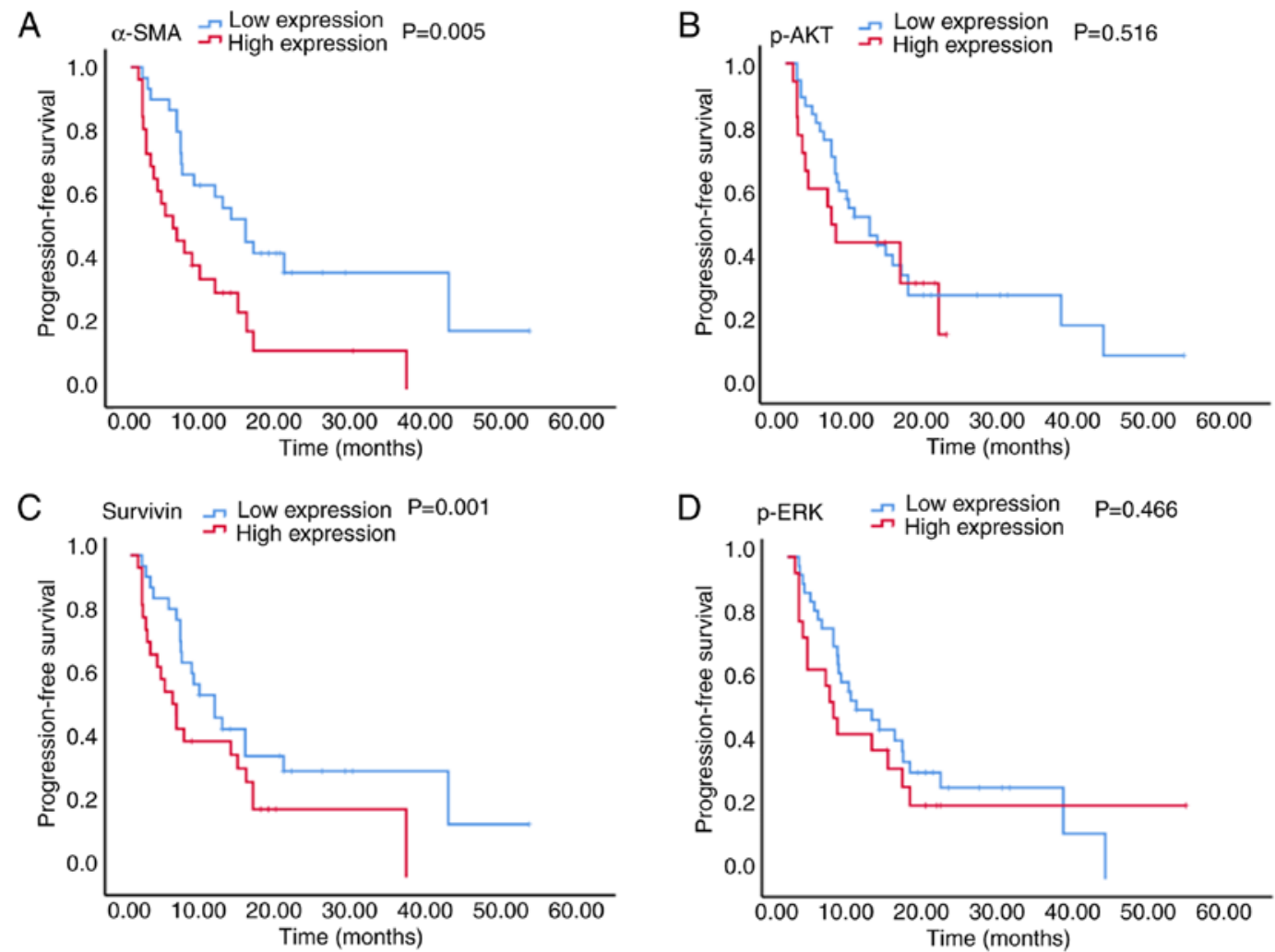

Figure 3. Kaplan-Meier survival curves for PFS in patients with stage IV colorectal cancer. (A) Patients with high $\alpha$-SMA expression exhibited a significantly reduced PFS time compared with that of patients with low $\alpha$-SMA expression (median PFS time, 5.5 vs. 15.0 months; P=0.005). (B) p-AKT and (D) p-ERK expression in these patients was not associated with PFS time. (C) Patients with high survivin expression exhibited a significantly reduced PFS time compared with that in patients with low survivin expression (median PFS time, 5.5 vs. 15.0 months; $\mathrm{P}=0.001$ ). $\alpha$-SMA, $\alpha$-smooth muscle actin; p-, phosphorylated; PFS, progression-free survival.
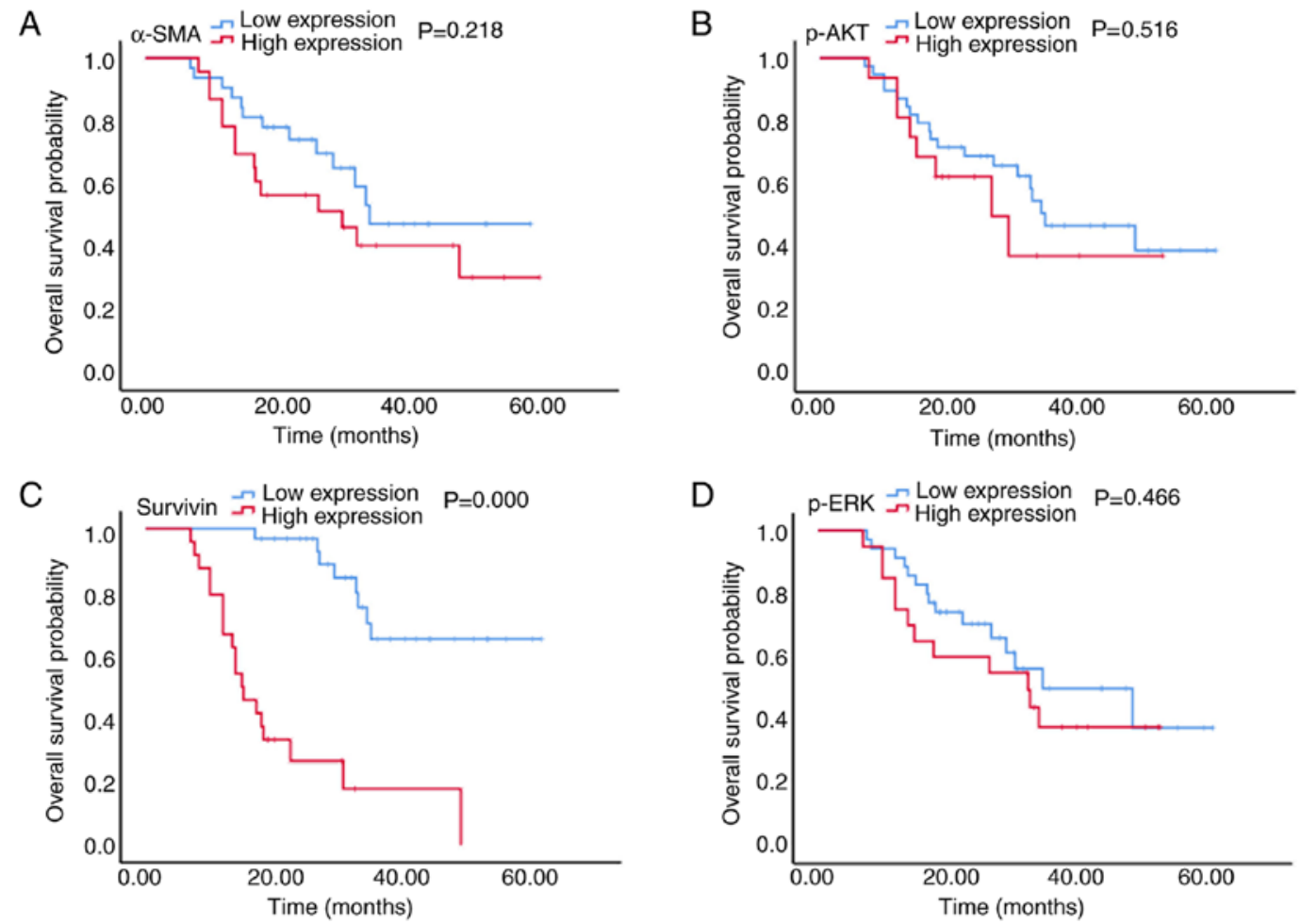

Figure 4. Kaplan-Meier survival curves for OS in patients with stage IV colorectal cancer. (A) Patients with high $\alpha$-SMA expression experienced a reduced OS time compared with that of patients with low $\alpha$-SMA expression, although this difference was not significant (median OS, 30.7 vs. 35.0 months; $\mathrm{P}=0.218$ ). (B) p-AKT and (D) p-ERK expression in these patients was not associated with OS time. (C) Patients with high survivin expression exhibited a significantly reduced OS time compared with that of patients with low survivin expression (median OS, 15.0 vs. 50.0 months, $\mathrm{P}<0.001$ ). $\alpha$-SMA, $\alpha$-smooth muscle actin; p-, phosphorylated; OS, overall survival. 
A

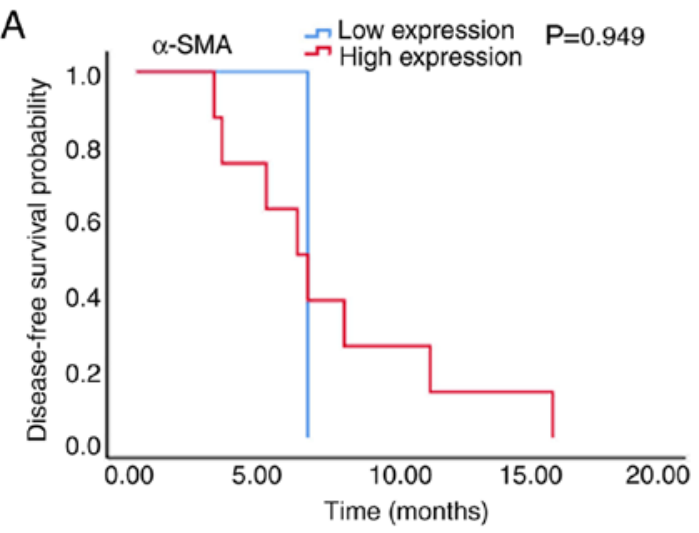

C

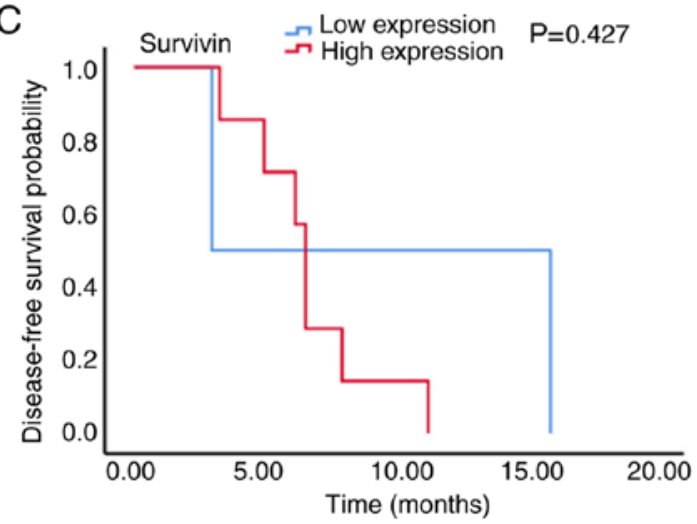

B

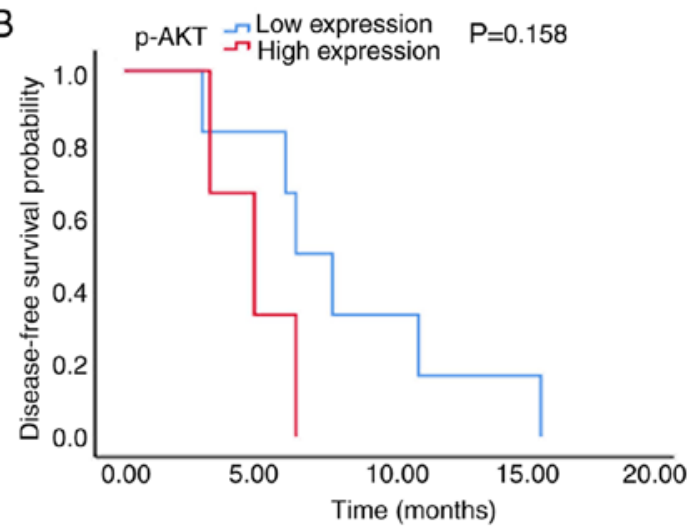

D

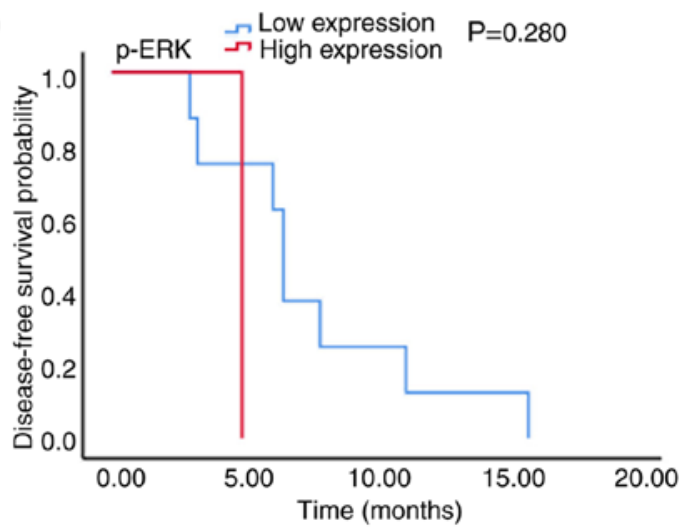

Figure 5. Kaplan-Meier survival curves for DFS in patients with stage III colorectal cancer. (A) $\alpha$-SMA, (B) p-AKT, (C) survivin and (D) p-ERK expression in stage III patients was not associated with DFS time. $\alpha$-SMA, $\alpha$-smooth muscle actin; p-, phosphorylated; DFS, disease-free survival.

A

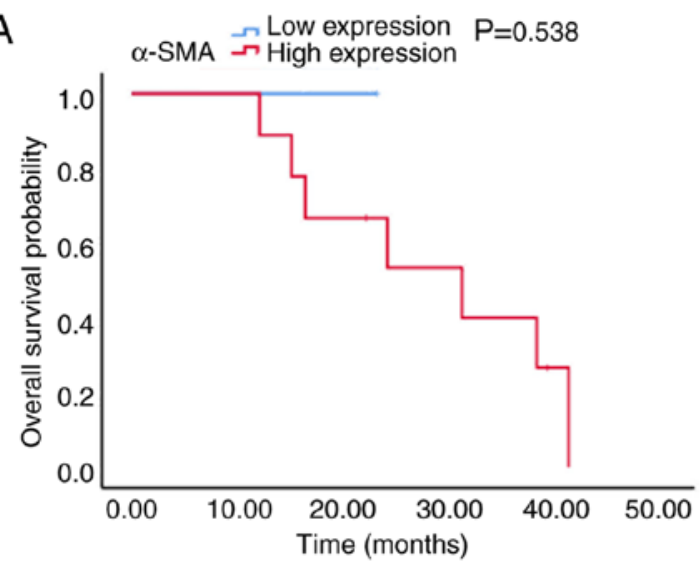

C

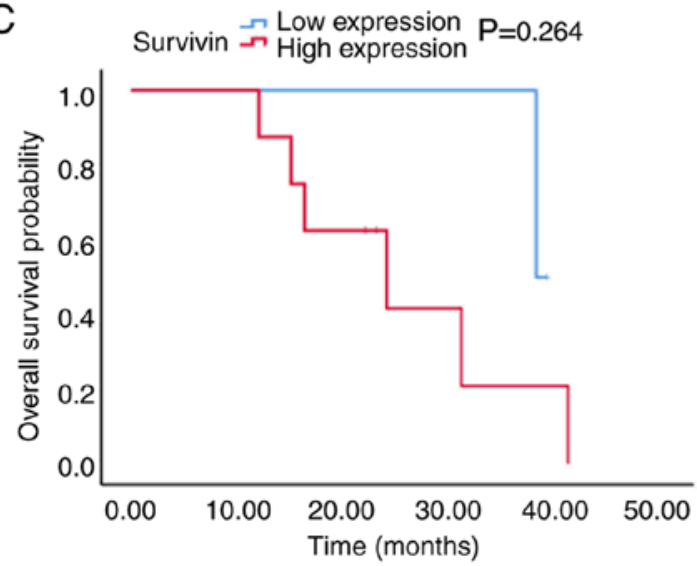

B

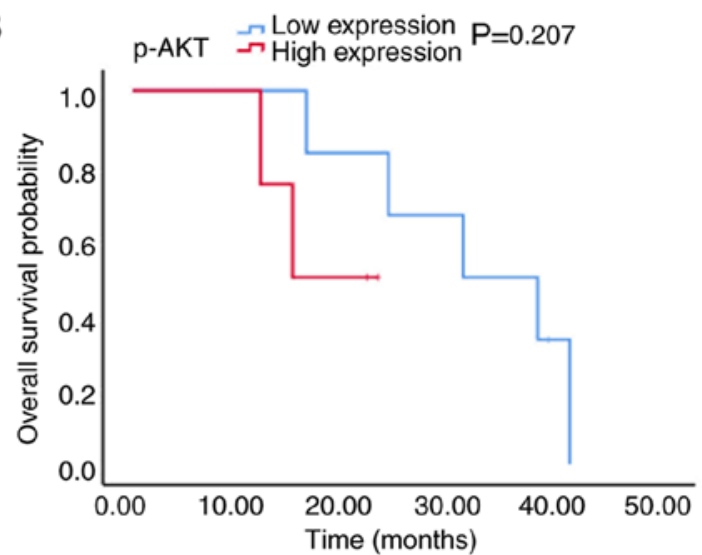

D

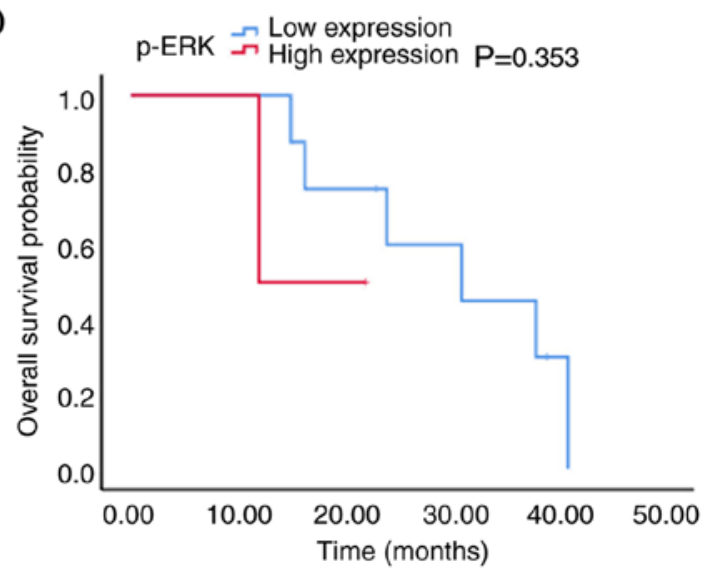

Figure 6. Kaplan-Meier survival curves for OS in patients with stage III colorectal cancer. (A) $\alpha$-SMA, (B) p-AKT, (C) survivin and (D) p-ERK expression in stage III patients was not associated with OS time. $\alpha$-SMA, $\alpha$-smooth muscle actin; p-, phosphorylated; OS, overall survival. 
Table II. Predictive potential of individual features for chemotherapy response in patients with advanced colorectal cancer. ${ }^{\mathrm{a}}$

\begin{tabular}{lcccc}
\hline Protein & $\begin{array}{c}\text { Classification of } \\
\text { each feature }\end{array}$ & $\begin{array}{c}\text { Cases of non-responders to feature } \\
\text { chemoeach each feature }\end{array}$ & $\begin{array}{c}\text { Cases of chemotherapy } \\
\text { responders, } \mathrm{n}\end{array}$ \\
\hline$\alpha$-SMA & 1 & 2 & 17 & 10 \\
p-AKT & 2 & 14 & 23 & 28 \\
& 1 & 11 & 16 & 35 \\
Survivin & 2 & 5 & 11 & 30 \\
p-ERK & 1 & 2 & 15 & 12 \\
\end{tabular}

${ }^{a}$ In the probabilistic neural networks model, the predictive potential of individual features were first analyzed. In each feature classification $1=$ low expression, $2=$ high expression. According to the probabilistic neural networks model, error rate $(\%)=$ error samples number/total number. The error rates $(\%)$ of each feature were as follows: $\alpha$-SMA $=(2+2+6+1+1) / 43=28 \%$; p-AKT $=(1+2+2+8+1+1) / 43=35 \%$; surviving $=(2+2+2+6+1) \div 43=30 \%$; p-ERK $=(6+2+2+2+8+1+1+1) / 43=53.5 \%$. $\alpha$-SMA, $\alpha$-smooth muscle actin; p-, phosphorylated.

Table III. Predictive potential of the combination of $\alpha$-SMA expression and survivin expression for chemotherapy response in patients with advanced colorectal cancer. ${ }^{\mathrm{a}}$

\begin{tabular}{lccc}
\hline $\begin{array}{l}\text { Classification of } \\
\alpha-S M A \text { expression }\end{array}$ & $\begin{array}{c}\text { Classification of survivin } \\
\text { expression }\end{array}$ & $\begin{array}{c}\text { Cases of non-responders to } \\
\text { chemotherapy, } \mathrm{n}\end{array}$ & $\begin{array}{c}\text { Cases of chemotherapy } \\
\text { responders, } \mathrm{n}\end{array}$ \\
\hline 1 & 1 & 0 & 13 \\
1 & 2 & 2 & 4 \\
2 & 1 & 2 & 3 \\
2 & 2 & 12 & 7
\end{tabular}

${ }^{a}$ In the probabilistic neural networks model, the predictive potential of the combination of $\alpha$-SMA expression and survivin expression was analyzed for chemotherapy response. In the classification of each feature ( $\alpha$-SMA expression or survivin expression), $1=$ low expression, $2=$ high expression. The number of non-responders to chemotherapy or chemotherapy responders in different combinations of $\alpha$-SMA expression and survivin expression is depicted in the table. Error rate $(\%)=$ error samples number/total number. Error rate $(\%)$ of these features was as follows: Error rate $(\%)=(2+2+6+1) / 43=26 \%$. $\alpha$-SMA, $\alpha$-smooth muscle actin.

Table IV. Predictive potential of the combination of $\alpha$-SMA, survivin and p-AKT expression for chemotherapy response in patients with advanced CRC.

\begin{tabular}{|c|c|c|c|c|c|}
\hline $\begin{array}{l}\text { Classification } \\
\text { of } \alpha \text {-SMA } \\
\text { expression }\end{array}$ & $\begin{array}{l}\text { Classification } \\
\text { of survivin } \\
\text { expression }\end{array}$ & $\begin{array}{l}\text { Classification } \\
\text { of p-AKT } \\
\text { expression }\end{array}$ & $\begin{array}{c}\text { Cases of } \\
\text { non-responders to } \\
\text { chemotherapy, } \mathrm{n}\end{array}$ & $\begin{array}{c}\text { Cases of } \\
\text { chemotherapy } \\
\text { responders, } n\end{array}$ & $\begin{array}{c}\text { Error rate, } \\
\%\end{array}$ \\
\hline 1 & 1 & 1 & 0 & 10 & 21 \\
\hline 1 & 1 & 2 & 0 & 4 & \\
\hline 1 & 2 & 1 & 0 & 3 & \\
\hline 1 & 2 & 2 & 2 & 0 & \\
\hline 2 & 1 & 1 & 2 & 2 & \\
\hline 2 & 1 & 2 & 9 & 7 & \\
\hline 2 & 2 & 1 & 0 & 1 & \\
\hline 2 & 2 & 2 & 3 & 0 & \\
\hline
\end{tabular}

In the probabilistic neural networks model, the predictive potential of the combination of $\alpha$-SMA, survivin and p-AKT expression was analyzed for chemotherapy response. In the classification of each feature ( $\alpha$-SMA expression, survivin expression and p-AKT expression), $1=$ low expression, 2=high expression. The number of non-responders to chemotherapy or chemotherapy responders in different combinations of $\alpha$-SMA expression, survivin expression and p-AKT expression was depicted in this table. Error rate $(\%)=$ error samples number/total number. Error rate $(\%)$ of these features was as follows: Error rate $(\%)=(2+7) / 43=21 \%$. $\alpha$-SMA, $\alpha$-smooth muscle actin; p-, phosphorylated. 
Table V. Predictive potential of $\alpha$-SMA, survivin, p-AKT and p-ERK expression for chemotherapy response in patients with advanced colorectal cancer. ${ }^{\mathrm{a}}$

\begin{tabular}{|c|c|c|c|c|c|c|}
\hline $\begin{array}{l}\text { Classification } \\
\text { of } \alpha \text {-SMA } \\
\text { expression }\end{array}$ & $\begin{array}{l}\text { Classification } \\
\text { of survivin } \\
\text { expression }\end{array}$ & $\begin{array}{l}\text { Classification } \\
\text { of p-AKT } \\
\text { expression }\end{array}$ & $\begin{array}{c}\text { Classification } \\
\text { of p-ERK } \\
\text { expression }\end{array}$ & $\begin{array}{l}\text { Cases of no } \\
\text { responders to } \\
\text { chemotherapy, } \mathrm{n}\end{array}$ & $\begin{array}{l}\text { Cases of } \\
\text { chemotherapy } \\
\text { responders, } n\end{array}$ & $\begin{array}{c}\text { Error rate, } \\
\%\end{array}$ \\
\hline 1 & 1 & 1 & 1 & 0 & 4 & 16 \\
\hline 1 & 1 & 1 & 2 & 0 & 6 & \\
\hline 1 & 1 & 2 & 1 & 0 & 2 & \\
\hline 1 & 1 & 2 & 2 & 0 & 2 & \\
\hline 1 & 2 & 1 & 1 & 0 & 1 & \\
\hline 1 & 2 & 1 & 2 & 0 & 2 & \\
\hline 1 & 2 & 2 & 1 & 2 & 0 & \\
\hline 1 & 2 & 2 & 2 & I & I & \\
\hline 2 & 1 & 1 & 1 & 0 & 2 & \\
\hline 2 & 1 & 1 & 2 & 2 & 0 & \\
\hline 2 & 1 & 2 & 1 & 8 & 6 & \\
\hline 2 & 1 & 2 & 2 & 1 & 1 & \\
\hline 2 & 2 & 1 & 1 & I & 1 & \\
\hline 2 & 2 & 1 & 2 & 0 & 1 & \\
\hline 2 & 2 & 2 & 1 & 1 & 0 & \\
\hline 2 & 2 & 2 & 2 & 2 & 0 & \\
\hline
\end{tabular}

${ }^{a}$ In the probabilistic neural networks model, the predictive potential of the combination of $\alpha$-SMA, survivin, p-AKT and p-ERK expression was analyzed for chemotherapy response. In classification of each feature ( $\alpha$-SMA expression, survivin expression, $\mathrm{p}$-AKT expression and p-ERK expression), 1=low expression, 2=high expression. The number of non-responders to chemotherapy or chemotherapy responders in different combinations of $\alpha$-SMA expression, survivin expression, p-AKT expression and p-ERK expression was depicted in this table. Error rate $(\%)=$ error samples number/total number; Error rate $(\%)$ of these feature was as follows: Error rate $(\%)=(6+1) / 43=16 \%$. $\alpha-\mathrm{SMA}$, $\alpha$-smooth muscle actin; p-, phosphorylated.

Table VI. Results of modeling, verification and prediction of 4 features for chemotherapy response in patients with advanced colorectal cancer.

\begin{tabular}{lcccc}
\hline Features & $\begin{array}{c}\text { Modeling } \\
\text { accuracy, } \%\end{array}$ & $\begin{array}{c}\text { Validation } \\
\text { accuracy, } \%\end{array}$ & $\begin{array}{c}\text { Prediction } \\
\text { accuracy, } \%\end{array}$ & $\begin{array}{c}\text { Total modeling } \\
\text { and validation, \% }\end{array}$ \\
\hline$\alpha$-SMA & 72.1 & 78.6 & 85.7 & 82.1 \\
$\alpha$-SMA, survivin & 72.1 & 78.6 & 85.7 & 82.1 \\
$\alpha$-SMA, survivin, p-AKT & 79.1 & 78.6 & 78.6 & 78.6 \\
$\alpha$-SMA, survivin, p-AKT, p-ERK & 83.7 & 92.9 & 85.7 & 89.3 \\
\hline
\end{tabular}

$\alpha$-SMA, $\alpha$-smooth muscle actin; p-, phosphorylated.

\section{Discussion}

The first-line chemotherapy used for patients with aCRC is an oxaliplatin plus 5-FU regimen; however, the response rate is limited. It is therefore necessary to identify biomarkers that could help oncologists to accurately select patients who can benefit from this specific regimen.

As illustrated in the 'seed and soil' hypothesis, supportive tumor stroma (the soil) is important for tumor cell (the seed) progression $(25,26)$. CAFs are large and spindle-shaped mesenchymalcells that represent the major components of tumor stroma. Numerous studies have demonstrated that CAFs serve crucial roles in cancer progression and resistance to chemotherapeutic agents $(27,28)$. However, the association between CAFs and traditional chemotherapy in CRC remains unclear. Previous studies reported that cytokines secreted by CAFs, including interleukin (IL)-8, IL-1 $\beta$, vascular endothelial growth factor, TNF- $\alpha$, IL-17 and IL- 6 , can predict a poor response to chemotherapy in CRC cells (29). Gonçalves-Ribeiro et al (11) revealed that CAFs have a protective effect on CRC cells. It has also been reported that the expression of CAFs-associated fibronectin 1 and collagen 3A1 can predict the absence of response to neoadjuvant treatment in locally advanced rectal carcinoma (30). The potential mechanisms involved are as follows: i) CAFs can secrete collagen type I, which can decrease chemotherapeutic drug uptake in tumors and then induce drug resistance $(31,32)$; 
ii) soluble factors secreted by CAFs can induce the activation of PI3K/AKT/survivin and JAK/STAT pathways, which may provide protection from cell death, ensure correct DNA repair and eventually induce resistance to oxaliplatin and 5-FU; and iii) chemotherapy-treated CAFs can secrete specific cytokines and chemokines, including IL-17A, which may promote cancer-initiating cell self-renewal and tumor resistance (33).

Increasing evidence has demonstrated that chemotherapy resistance may be associated with the activation of two important Ras downstream pathways, the MAPK and the PI3K-AKT-mTOR signaling pathways (12-14). The MAPK pathway regulates numerous physiological processes in cancer cells, including cell proliferation, metastasis and chemoresistance (34). Previous studies revealed that MAPK signaling pathway activation serves key roles in drug resistance, notably in gastric cancer, breast cancer, prostate cancer and CRC. The PI3K/AKT/mTOR pathway also regulates cancer progression and is recognized as a major cause of multidrug resistance in various types of cancer (15-18). This pathway has also been identified as a potent contributor to drug resistance in CRC, particularly resistance to 5-FU and oxaliplatin (35). Inhibition of the PI3K/AKT signaling pathway can reduce insulin-induced chemotherapeutic drug resistance. AKT phosphorylation serves a pivotal part in the activation of the $\mathrm{PI} 3 \mathrm{~K} / \mathrm{AKT} / \mathrm{mTOR}$ signaling pathway $(36,37)$.

Survivin is a member of the inhibitor of apoptosis proteins family. Numerous studies have demonstrated that survivin overexpression is associated with drug resistance in different cancer types (19,38-40). The potential mechanisms involved in survivin overexpression-induced drug resistance are as follows: i) Nuclear survivin serves a pivotal role in the formation of spindle fiber assembly via stabilization of microtubule formation leading to cell growth (41); ii) survivin can upregulate the molecular sensor of DNA damage named ku70 and subsequently enhance the repair of DNA double-stranded breaks (42); and iii) survivin can interact with the apoptosis-inducing molecules caspase- 3 and casapase-9 and inhibit their apoptotic function (43). Additional evidence has revealed that regulation of survivin expression may be a downstream effect of the MAPK (44-46) or PI3K-AKT-mTOR signaling pathways (45) in different types of cancer.

Based on this evidence, the present study established a predictive model of $\alpha$-SMA, p-AKT, p-ERK and survivin expression in patients with CRC in order to anticipate the potential intrinsic resistance to the oxaliplatin plus 5-FU regimen. The results from this study demonstrated that $\alpha$-SMA and survivin overexpression in patients with CRC was significantly associated with oxaliplatin plus 5-FU resistance, which was not the case for p-AKT and p-ERK overexpression; this result was in agreement with previous studies and hypotheses (19,43-45). From these different patterns, the model comprising $\alpha$-SMA, p-AKT, p-ERK and survivin overexpression was the best predictive pattern. The predictive model of $\alpha$-SMA, p-AKT, p-ERK and survivin overexpression was of great efficacy (81.3\%) and accuracy (81.8\%), and could aid oncologists in determining whether an individual could benefit from the oxaliplatin plus 5-FU chemotherapy regimen.

In conclusion, the multifactorial predictive biomarker model of $\alpha$-SMA, p-AKT, p-ERK and survivin expression in patients with CRC used to predict intrinsic resistance to the oxaliplatin plus 5-FU regimen in the present study was efficient and accurate. These results suggested that patients with high expression of this predictive model may be intrinsically resistant to an oxaliplatin plus 5-FU regimen. This predictive model may be of great help to screen cancer patients who have intrinsic chemoresistance and to prescribe them personalized treatments.

\section{Acknowledgements}

Not applicable.

\section{Funding}

This study was supported by the National Natural Science Foundation of China (grant nos. 81472785 and 61435001) and the CA MS Innovation Fund for Medical Sciences (grant no. 2016-12M-1-001).

\section{Availability of data and materials}

The datasets used and/or analyzed during the current study are available from the corresponding author on reasonable request.

\section{Authors' contributions}

JG made substantial contributions to conception and design, acquisition of data, analysis and interpretation of data and drafting of the manuscript. ZL made substantial contributions to acquisition of data. JZ made substantial contributions to conception and design of the study and acquisition of data. ZS made substantial contributions to conception and design, analysis and interpretation of data, drafting the manuscript and revising it critically for important intellectual content. CB made substantial contributions to conception and design, interpretation of data, and revised the manuscript critically for important intellectual content. All authors read and approved the final manuscript.

\section{Ethics approval and consent to participate}

This study was performed in accordance with the Declaration of Helsinki and was approved by the Peking Union Medical College Hospital Ethics Committee (no. ZS-1358). All patients were informed about the use of their tissue samples for biological research and provided written informed consent prior to the study.

\section{Patient consent for publication}

Not applicable.

\section{Competing interests}

The authors declare that they have no competing interests.

\section{References}

1. Siegel RL, Miller KD and Jemal A: Cancer statistics, 2015. CA Cancer J Clin 65: 5-29, 2015.

2. Kap EJ, Seibold P, Richter S, Scherer D, Habermann N, Balavarca Y, Jansen L, Becker N, Pfütze K, Popanda O, et al: Genetic variants in DNA repair genes as potential predictive markers for oxaliplatin chemotherapy in colorectal cancer. Pharmacogenomics J 15: 505-512, 2015. 
3. Napolitano S, Martini G, Rinaldi B, Martinelli E, Donniacuo M, Berrino L, Vitagliano D, Morgillo F, Barra G, De Palma R, et al: Primary and acquired resistance of colorectal cancer to anti-EGFR monoclonal antibody can be overcome by combined treatment of regorafenib with cetuximab. Clin Cancer Res 21: 2975-2983, 2015

4. Ye F, Liu Z, Tan A, Liao M, Mo Z and Yang X: XRCC1 and GSTP1 polymorphisms and prognosis of oxaliplatin-based chemotherapy in colorectal cancer: A meta-analysis. Cancer Chemother Pharmacol 71: 733-740, 2013.

5. Noda E, Maeda K, Inoue T, Fukunaga S, Nagahara $H$, Shibutani M, Amano R, Nakata B, Tanaka H, Muguruma K, et al Predictive value of expression of ERCC 1 and GST-p for 5-fluorouracil/oxaliplatin chemotherapy in advanced colorectal cancer. Hepatogastroenterology 59: 130-133, 2012.

6. Chen J, Xie F, Chen K, Wang D, Jiang H, Li J, Pan F, Chen S, Zhang Y, Ruan Z, et al: ERCC5 promoter polymorphisms at -763 and +25 predict the response to oxaliplatin-based chemotherapy in patients with advanced colorectal cancer. Cancer Biol Ther 8: 1424-1430, 2009.

7. Fariña-Sarasqueta A, van Lijnschoten $G$, Rutten $\mathrm{HJ}$ and van den Brule AJ: Value of gene polymorphisms as markers of 5-FU therapy response in stage III colon carcinoma: A pilot study. Cancer Chemother Pharmacol 66: 1167-1171, 2010.

8. Li S, Lu X, Chi P and Pan J: Identification of Nkx2-3 and TGFB1I1 expression levels as potential biomarkers to predict the effects of FOLFOX4 chemotherapy. Cancer Biol Ther 13: 443-449, 2012.

9. Li S, Lu X, Chi P and Pan J: Identification of HOXB8 and KLK11 expression levels as potential biomarkers to predict the effects of FOLFOX4 chemotherapy. Future Oncol 9: 727-736, 2013

10. Schetter AJ, Leung SY, Sohn JJ, Zanetti KA, Bowman ED, Yanaihara N, Yuen ST, Chan TL, Kwong DL,Au GK, et al: MicroRNA expression profiles associated with prognosis and therapeutic outcome in colon adenocarcinoma. JAMA 299: 425-436, 2008.

11. Gonçalves-Ribeiro S, Díaz-Maroto NG, Berdiel-Acer M, Soriano A, Guardiola J, Martínez-Villacampa M, Salazar R, Capellà G, Villanueva A, Martínez-Balibrea E and Molleví DG: Carcinoma-associated fibroblasts affect sensitivity to oxaliplatin and 5FU in colorectal cancer cells. Oncotarget 7: 59766-59780, 2016.

12. WestKA,CastilloSS andDennisPA:Activation ofthePI3K/Aktpathway and chemotherapeutic resistance. Drug Resist Update 5: 234-248, 2002.

13. McCubrey JA, Steelman LS, Chappell WH, Abrams SL, Wong EW, Chang F, Lehmann B, Terrian DM, Milella M, Tafuri A, et al: Roles of the RAF/MEK/ERK pathway in cell growth, malignant transformation and drug resistance. Biochim Biophys Acta 1773: 1263-1284, 2007.

14. Pritchard AL and Hayward NK: Molecular pathways: mitogen-activated protein kinase pathway mutations and drug resistance. Clin Cancer Res 19: 2301-2309, 2013.

15. Zhao H, Zhao D, Jin H, Li H, Yang X, Zhuang L and Liu T: Bufalin reverses intrinsic and acquired drug resistance to cisplatin through the AKT signaling pathway in gastric cancer cells. Mol Med Rep 14: 1817-1822, 2016.

16. LoPiccolo J, Blumenthal GM, Bernstein WB and Dennis PA: Targeting the PI3K/Akt/mTOR pathway: Effective combinations and clinical considerations. Drug Resist Updat 11: 32-50, 2008.

17. Sun L, Liu L, Liu X, Wang Y, Li M, Yao L, Yang J, Ji G, Guo C Pan Y, et al: MGrl-Ag/37LRP induces cell adhesion-mediated drug resistance through FAK/PI3K and MAPK pathway in gastric cancer. Cancer Sci 105: 651-659, 2014.

18. Jin W, Wu L, Liang K, Liu B, Lu Y and Fan Z: Roles of the PI-3K and MEK pathways in Ras-mediated chemoresistance in breast cancer cells. Br J Cancer 89: 185-191, 2003.

19. Nam K, Son SH, Oh S, Jeon D, Kim H, Noh DY, Kim S and Shin I: Binding of galectin-1 to integrin $\beta 1$ potentiates drug resistance by promoting survivin expression in breast cancer cells. Oncotarget 8: 35804-35823, 2017.

20. Hari DM, Leung AM, Lee JH, Sim MS, Vuong B, Chiu CG and Bilchik AJ: AJCC Cancer Staging Manual 7th edition staging criteria for colon cancer: do the complex modifications improve prognostic assessment? J Am Coll Surg 217: 181-190, 2013.

21. Benson AB III, Venook AP, Cederquist L, Chan E, Chen YJ, Cooper HS, Deming D, Engstrom PF, Enzinger PC, Fichera A, et al: Colon cancer, version 1.2017, NCCN clinical practice guidelines in oncology. J Natl Compr Canc Netw 15: 370-398, 2017.

22. Eisenhauer EA, Therasse P, Bogaerts J, Schwartz LH, Sargent D, Ford R, Dancey J, Arbuck S, Gwyther S, Mooney M, et al: New response evaluation criteria in solid tumours: Revised RECIST guideline (version 1.1). Eur J Cancer 45: 228-247, 2009.

23. Cai J, Guan H, Fang L, Yang Y, Zhu X, Yuan J, Wu J and Li M MicroRNA-374a activates Wnt/ $\beta$-catenin signaling to promote breast cancer metastasis. J Clin Invest 12: 566-579, 2013
24. Spech DF: Probabilistic neural networks. Neural Networks 3: $109-118,1990$.

25. Mueller MM and Fusenig NE: Friends or foes-bipolar effects of the tumour stroma in cancer. Nat Rev Cancer 4: 839-849, 2004.

26. Paget S: The distribution of secondary growths in cancer of the breast. 1989. Cancer Metastasis Rev 8: 98-101, 1889.

27. Mei L, Du W and Ma WW: Targeting stromal microenvironment in pancreatic ductal adenocarcinoma: Controversies and promises. J Gastrointest Oncol 7: 487-494, 2016

28. Rachel JB and Sun YO: Breast cancer-associated fibroblasts: Where we are and where we need to go. Cancers (Basel) 8: pii:E19, 2016.

29. Nagasaki T, Hara M, Nakanishi H, Takahashi H, Sato $M$ and Takeyama H: Interleukin- 6 released by colon cancer-associated fibroblasts is critical for tumour angiogenesis: Anti-interleukin-6 receptor antibody suppressed angiogenesis and inhibited tumour-stroma interaction. Br J Cancer 110: 469-478, 2014

30. Goncalves-Ribeiro S, Sanz-Pamplona R, Vidal A, Sanjuan X, Guillen Díaz-Maroto N, Soriano A, Guardiola J, Albert N, Matinez-Villacampa M, Lopez I, et al: Prediction of pathological response to neoadjuvant treatment in rectal cancer with a two-protein immunohistochemical score derived from stromal gene-profiling. Ann Oncol 28: 2160-2168, 2017.

31. Loeffler M, Krüger JA, Niethammer AG and Reisfeld RA: Targeting tumor-associated fibroblasts improves cancer chemotherapy by increasing intratumoral drug uptake. J Clin Invest 116: 1955-1962, 2006

32. Mao Y, Keller ET, Garfield DH, Shen K and Wang J: Stromal cells in tumor microenvironment and breast cancer. Cancer Metastasis Rev 32: 303-315, 2013.

33. Lotti F, Jarrar AM, Pai RK, Hitomi M, Lathia J, Mace A, Gantt GA Jr, Sukhdeo K, DeVecchio J, Vasanji A, et al: Chemotherapy activates cancer-associated fibroblasts to maintain colorectal cancer-initiating cells by IL-17A. J Exp Med 210: 2851-2872, 2013

34. Das Thakur M and Stuart DD: Molecular pathways: Response and resistance to BRAF and MEK inhibitors in BRAF(V600E) tumors. Clin Cancer Res 20: 1074-1080, 2014.

35. Chen J, Shao R, Li F, Monteiro M, Liu JP, Xu ZP and Gu W: PI3K/Akt/mTOR pathway dual inhibitor BEZ235 suppresses the stemness of colon cancer stem cells. Clin Exp Pharmacol Physiol 42: 1317-1326, 2015.

36. Xu J, Zhang SR, Wang R, Wu X, Zeng L and Fu Z: Knockdown of PRDX2 sensitizes colon cancer cells to 5-FU by suppressing the PI3K/AKT signaling pathway. Biosci Rep 37: pii: BSR20160447, 2017.

37. Danielsen SA, Eide PW, Nesbakken A, Guren T, Leithe E and Lothe RA: Portrait of the PI3K/AKT pathway in colorectal cancer. Biochim Biophys Acta 1855: 104-121, 2015.

38. Cheung CH, Huang CC, Tsai FY, Lee JY, Cheng SM, Chang YC, Huang YC, Chen SH and Chang JY: Survivin-biology and potential as a thera-peutic target in oncology. Onco Targets Ther 6: 1453-1462, 2013.

39. Zaffaroni N and Daidone MG: Survivin expression and resistance to anticancer treatments: Perspectives for new therapeutic interventions. Drug Resist Updat 5: 65-72, 2002.

40. Coumar MS, Tsai FY, Kanwar JR, Sarvagalla S and Cheung CH: Treat cancers by targeting survivin: Just a dream or future reality? Cancer Treat Rev 39: 802-811, 2013.

41. Li F, Yang J, Ramnath N, Javle MM and Tan D: Nuclear or cytoplasmic expression of survivin: What is the significance? Int J Cancer 114: 509-512, 2005.

42. Jiang G, Ren B, Xu L, Song S, Zhu C and Ye F: Survivin may enhance DNA double-strand break repair capability by up-regulating Ku70 in human KB cells. Anticancer Res 29: 223-228, 2009.

43. Shin S, Sung BJ, Cho YS, Kim HJ, Ha NC, Hwang JI, Chung CW, Jung YK and Oh BH: An anti-apoptotic protein human survivin is a direct inhibitor of caspase-3 and -7. Biochemistry 40: 1117-1123, 2001

44. Zhuo W, Zhang L, Zhu Y, Zhu B and Chen Z: Fisetin, a dietary bioflavonoid, reverses acquired Cisplatin-resistance of lung adenocarcinoma cells through MAPK/Survivin/Caspase pathway. Am J Transl Res 7: 2045-2052, 2015.

45. Tsubaki M, Takeda T, Ogawa N, Sakamoto K, Shimaoka H, Fujita A, Itoh T, Imano M, Ishizaka T, Satou T and Nishida S: Overexpression of survivin via activation of ERK1/2, Akt, and $\mathrm{NF}-\mathrm{kB}$ plays a central role in vincristine resistance in multiple myeloma cells. Leuk Res 39: 445-452, 2015.

46. Zhang Y, Chen HX, Zhou SY, Wang SX, Zheng K, Xu DD, Liu YT, Wang XY, Wang X, Yan HZ, et al: Sp1 and c-Myc modulate drug resistance of leukemia stem cells by regulating survivin expression through the ERK-MSK MAPK signaling pathway. Mol Cancer 14: 56, 2015. 